\title{
TEST WORDS OF A FREE PRODUCT OF TWO FINITE CYCLIC GROUPS
}

\author{
by DANIEL A. VOCE
}

(Received 11th December 1995)

We characterize the test words of $\mathbb{Z}_{m} * \mathbb{Z}_{n}$. They are those elements not contained in a proper retract.

1991 Mathematics subject classification: 20E05, 20F55.

\section{Introduction}

An element $w$ of a group $\Gamma$ is a test element if every endomorphism of $\Gamma$ fixing $w$ is necessarily an automorphism. If $\Gamma$ is a free group or a free product then the test elements are called test words. The element $w$ is called a test element for monomorphisms if every monomorphism of $\Gamma$ fixing $w$ is necessarily an automorphism. Given a test element $w$, the endomorphism $\phi$ is an automorphism if and only if $\phi(w)=\alpha(w)$ for some automorphism $\alpha$. Thus, the use of test elements provides a method for recognizing automorphisms of a particular group. In what follows we prove these results (terminology explained in Section 2).

Theorem 1. If $G=\mathbb{Z}_{m} * \mathbb{Z}_{n}$ and $\phi$ is a monomorphism of $G$ then the stable image of $\phi$ is a free factor of $G$.

Corollary 2. The test words for monomorphisms of $G$ are those words of infinite order-i.e., those words not lying in a proper free factor.

Theorem 2. If $G=\mathbb{Z}_{m} * \mathbb{Z}_{n}$ and $\phi$ is an endomorphism of $G$ then the stable image of $\phi$ is a retract of $G$.

Corollary 3. The test words of $G$ are those words not lying in a proper retract.

Specific examples of test words in a free product of two finite cyclic groups are given in Section 5. It should be noted that Turner [4] has proven the results listed above for the case when $G$ is a finitely generated free group. I would like to acknowledge his contribution to this work as my dissertation advisor. 


\section{Preliminaries}

Definition 1. [1] If $\phi: \Gamma \rightarrow \Gamma$ is an endomorphism of an arbitrary group $\Gamma$ then the stable image of $\phi$ is

$$
\phi^{\infty}(\Gamma)=\bigcap_{i=1}^{\infty} \phi^{i}(\Gamma), \quad \text { and } \quad \phi_{\infty}=\phi \mid \phi^{\infty}(\Gamma) .
$$

We shall see that the stable image plays an important part in our investigation of test elements. Suppose that $w$ is a test element in a group $\Gamma$. Then $w$ may not lie in a proper retract of $\Gamma$ since otherwise, there would be a non-automorphism fixing $w$. Conversely, if $w$ is not a test element then there exists an endomorphism $\phi: \Gamma \rightarrow \Gamma$ fixing $w$ so that $\phi$ is not an automorphism. If $\Gamma$ is Hopfian then $\phi$ cannot be a surjection and $\phi^{\infty}(\Gamma)$ is a proper subgroup containing $w$. We shall exhibit groups in which $\phi^{\infty}(\Gamma)$ is actually a proper retract containing $w$.

As motivation, we first examine the stable image of an endomorphism of a finite group $T$ and provide a retract characterization for test words of $T$. Recall that a group satisfies the ascending chain condition on subgroups (ACC) if every ascending chain of subgroups eventually stabilizes. Clearly every finite group satisfies the ACC.

Lemma 1. If $\phi: \Gamma \rightarrow \Gamma$ and $\Gamma$ satisfies the $A C C$ then $\phi_{\infty}$ is an automorphism.

Proof. Consider the chain of maps

$$
\Gamma \stackrel{\phi_{1}}{\longrightarrow} \phi(\Gamma) \stackrel{\phi_{2}}{\longrightarrow} \phi^{2}(\Gamma) \rightarrow \ldots \rightarrow \phi^{k-1}(\Gamma) \stackrel{\phi_{k}}{\longrightarrow} \phi^{k}(\Gamma) \rightarrow \ldots
$$

where $\phi_{k}$ is the restriction of $\phi$ to the subgroup $\phi^{k-1}(\Gamma)$. Let $\psi_{k}=\phi_{k} \phi_{k-1} \ldots \phi_{1}$ : $\Gamma \rightarrow \phi^{k}(\Gamma)$. We have an ascending chain of subgroups $\operatorname{ker}\left(\psi_{1}\right)<\operatorname{ker}\left(\psi_{2}\right)<\ldots<\Gamma$ so there exists an $N$ such that $\operatorname{ker}\left(\psi_{k}\right)=\operatorname{ker}\left(\psi_{N}\right)$ for every $k \geq N$. This shows that the maps $\phi_{k}$ are eventually injective and hence $\phi_{\infty}$ is also injective. We now show that $\phi_{\infty}$ is surjective.

If $g \in \phi^{\infty}(\Gamma)$ then $g=\phi^{n}\left(g_{n}\right)$ for every $n$ and for some $g_{n} \in \Gamma$. Choose $N$ so that $\phi_{N}: \phi^{N-1}(\Gamma) \rightarrow \phi^{N}(\Gamma)$ is injective. For $n \geq N$ the elements $\phi^{n-1}\left(g_{n}\right)$ are in the subgroup $\phi^{N-1}(\Gamma)$. Furthermore, $\phi_{N}\left(\phi^{n-1}\left(g_{n}\right)\right)=g$ and by injectivity we get the equations

$$
\phi^{N-1}\left(g_{N}\right)=\phi^{N}\left(g_{N+1}\right)=\phi^{N+1}\left(g_{N+2}\right)=\ldots
$$

which means that $\phi^{N-1}\left(g_{N}\right) \in \phi^{\infty}(\Gamma)$ and that $\phi_{\infty}\left(\phi^{N-1}\left(g_{N}\right)\right)=\phi^{N}\left(g_{N}\right)=g$.

Proposition 1. If $T$ is a finite group then $w \in T$ is a test element if and only if $w$ does not lie in a proper retract of $T$.

Proof. Suppose that $w$ is not a test element and that $\phi$ is a non-automorphism fixing $w$. Since $T$ is finite there exists an $N$ such that $\phi^{k}(T)=\phi^{N}(T)$ for all $k \geq N$. 
Thus $\phi^{\infty}(T)=\phi^{N}(T)$ giving a retraction

$$
T \stackrel{\phi^{N}}{\longrightarrow} \phi^{N}(T) \stackrel{i d}{\longrightarrow} \phi^{\infty}(T) \stackrel{\left(\phi_{-1}^{-1}\right)^{N}}{\longrightarrow} \phi^{\infty}(T) .
$$

Since $\phi$ is not surjective, $\phi^{\infty}(T)$ is a proper retract of $T$ containing $w$.

If $\Gamma$ is any group containing a test element $w$ then $w$ cannot lie in a proper retract of $\Gamma$; in particular, the cyclic subgroup $(w)$ cannot be a proper retract. The next proposition shows how to decide if a given element generates such a retract. We denote the exponent sum of an element $w$ on a generator $x_{j}$ by $|w|_{x j}$.

Proposition 2. Suppose that $\Gamma$ has the presentation

$$
\Gamma=\left\langle x_{1}, x_{2}, \ldots, x_{s} \mid r_{1}, r_{2}, \ldots, r_{1}\right\rangle
$$

and $w \in \Gamma$. Let $|R|_{X}$ denote the $t \times s$ matrix whose $i j^{\text {th }}$ entry is $\left|r_{i}\right|_{x j}$ and let $|w|_{X}$ denote the $1 \times s$ exponent sum vector of $w$ on the generators $x_{j}$. Then $w$ generates a retract of $\Gamma$ if and only if there exists a solution to the equation:

$$
\left(\begin{array}{c}
|R|_{X} \\
-\overline{|w|_{X}}
\end{array}\right)\left(\begin{array}{c}
k_{1} \\
\vdots \\
k_{s}
\end{array}\right)=\left(\begin{array}{c}
0 \\
\vdots \\
0 \\
1
\end{array}\right) .
$$

If $w$ has finite order then this is an equation over $\mathbb{Z}_{n}$ where $n$ is the order of $w$, otherwise it is over $\mathbb{Z}$ and $n=0$.

Proof. Suppose that $\rho: \Gamma \rightarrow\langle w\rangle$ by $\rho\left(x_{i}\right)=w^{k_{i}}$. Then $r_{j}$ is mapped to $w^{l_{j}}$ where $l_{j}=k_{1}\left|r_{j}\right|_{x_{1}}+\ldots+k_{s}\left|r_{j}\right|_{x_{j}}$. Since $\rho$ is a homomorphism, $l_{j}=0(\bmod n)$. The element $w$ is mapped to $w^{l}$ where $l=k_{1}|w|_{x_{1}}+\ldots+k_{s}|w|_{x_{1}}$. Since $\rho$ is a retraction, $l=1(\bmod n)$. This argument reverses proving the converse.

Corollary 1. Suppose that $G$ is a quotient of the free group of finite rank $F(X)$ admitting the presentation $G=\langle X \mid R\rangle$ where $R \subset[F, F]$. If $w \in G$ has infinite order then $w$ generates a retract of $G$ if and only if the entries of $|w|_{X}$ are relatively prime.

Example 1. Suppose that $\left.T=\left\langle x_{1}, x_{2}\right| x_{1}^{2}, x_{2}^{8},\left[x_{1}, x_{2}\right]\right)$. Since $T$ is abelian the retracts of $T$ are precisely the direct factors of $T$. Any proper direct factor of $T$ is cyclic (this is, in general, not true for any finite abelian group of rank 2). We first check which elements of $T$ generate proper retracts. Choose $w \in T$ and suppose that $w=x_{1}^{s} x_{2}^{t}$ where $s \in \mathbb{Z}_{2}$ and $t \in \mathbb{Z}_{8}$. If $s=0$ or $t=0$ then $w$ lies in a proper retract so we may ignore these cases. By Proposition 2, $w$ generates a retract if and only if there exists a solution vector $K$ over $\mathbb{Z}_{|w|}$ to the equation 


$$
\left(\begin{array}{ll}
2 & 0 \\
0 & 8 \\
0 & 0 \\
s & t
\end{array}\right) \mathrm{K}=\left(\begin{array}{l}
0 \\
0 \\
0 \\
1
\end{array}\right)
$$

This happens if and only if $w \neq x_{1} x_{2}^{2}$ and $w \neq x_{1} x_{2}^{6}$. These are the possible test elements of $T$. But since these elements are proper powers of only each other then neither lies in a proper retract. Thus, these elements are precisely the test elements of $T$.

\section{Test words for monomorphisms of $\mathbb{Z}_{m} * \mathbb{Z}_{n}$}

For the remainder of this note $G$ will denote the group $\mathbb{Z}_{m} * \mathbb{Z}_{n}$ given the presentation

$$
G=\left\langle x, y \mid x^{m}, y^{n}\right\rangle
$$

Any element $w$ of $G$ is defined by a unique reduced word

$$
w=x^{a_{1}} y^{b_{1}} x^{a_{2}} y^{b_{2}} \ldots x^{a_{r}} y^{b_{r}}
$$

where the integers $a_{i}, b_{j}$ are reduced modulo $m$ and $n$ respectively. All exponents are nonzero except possibly $a_{1}$ and $b_{r}$. The length of $w$, denoted $|w|$, is the number of nonzero powers of generators appearing in its reduced form. For example, in the group $\mathbb{Z}_{4} * \mathbb{Z}_{13}=\left\langle x, y \mid x^{4}, y^{13}\right\rangle$ the length of $x^{2} y^{6}$ is 2 and the length of $y^{12} x^{3} y^{-1}$ is 3 .

By the Normal Form Theorem for free products, the only elements of finite order in $G$ are conjugates of powers of the generators $x$ and $y$. Because of this, any endomorphism $\phi$ of $G$ has one of the following four forms:
(1) $x \mapsto g x^{k} g^{-1}$
(2) $x \mapsto g x^{k} g^{-1}$
(3) $x \mapsto g y^{k} g^{-1}$
(4) $x \mapsto g y^{k} g^{-1}$
$y \mapsto h y^{\prime} h^{-1}$
$y \mapsto h x^{\prime} h^{-1}$
$y \mapsto h y^{l} h^{-1}$
$y \mapsto h x^{\prime} h^{-1}$

where $g$ and $h$ are arbitrary elements of $G$. We call the endomorphism in the $i^{\text {th }}$ column a type $i$ endomorphism, $1 \leq i \leq 4$.

Our main concern will be to prove that $\phi^{\infty}(G)$ is a retract of $G$. The previous paragraph suggests a proof of this result by using a case by case analysis on the conjugators $g$ and $h$. This is in fact our approach. Arguments for type 3 maps are analogous to those for type 2 maps so we will omit mention of type 3 maps in our proofs. Notice that if $\phi$ is a type 4 map then $\phi^{2}$ is type 1 . From the definition of the stable image $\phi^{\infty}(G)$ it is clear that $\phi^{\infty}(G)=\left(\phi^{2}\right)^{\infty}(G)$. Thus to prove that the stable image is a retract we need only concern ourselves with type 1 and type 2 endomorphisms.

Theorem 1. If $G=\left\langle x, y \mid x^{m}, y^{n}\right\rangle$ and $\phi$ is a monomorphism of $G$ then the stable image of $\phi$ is a free factor of $G$. 
Proof. By previous comments we can assume that $\phi(x)$ is a conjugate of $x^{k}$ and $\phi(y)$ is a conjugate of $y^{l}$ or $x^{l}$ for some $k$ and $l$. Since $\phi$ is injective, $k$, is relatively prime to $m$ (otherwise $\phi\left(x^{r}\right)=1$ where $r$ is the order of $x^{k}$ ). But then $x^{k}$ is an element of the multiplicative group of units of $\mathbb{Z}_{m}$ and $k^{s}=1(\bmod m)$ for some $s$. Hence $\phi^{s}(x)$ is a conjugate of $x$. Again, since $\phi^{\infty}(G)=\left(\phi^{3}\right)^{\infty}(G)$ we may assume that $\phi$ has the form

$$
\phi(x)=g x g^{-1}
$$

As for the image of $y$, if it is a conjugate of a power of $y$ then we may assume as we did for $x$ that

$$
\phi(y)=h y h^{-1} .
$$

To summarize thus far, we need only consider maps $\phi$ of one of the following types:

$$
\text { (1) } \begin{array}{rlrl}
x & \mapsto g x g^{-1} & \text { (2) } x & \mapsto g x g^{-1} \\
y & \mapsto h y h^{-1} & y & \mapsto h x^{\prime} h^{-1}
\end{array}
$$

In determining the structure of the stable image $\phi^{\infty}(G)$ we will first consider the map in the first column (a type 1 monomorphism). For this, we will need to consider several different possibilities for $g$ and $h$. In each case we will find that the stable image is either trivial, the entire group $G$, or a proper free factor. Type 2 monomorphisms will be considered after that.

Case 1: The map $\phi$ is a type 1 monomorphism, $g=1, h \neq 1$

$$
x \mapsto x \quad y \mapsto h y h^{-1}
$$

Clearly we may assume that $h$ does not end in a power of $y$. Also, if $h \in\langle x\rangle$ then $\phi$ is an inner automorphism and the stable image is $G$. Thus $h=\alpha y x^{k}$ or $h=\beta y^{-1} x^{k}$ where $\alpha$ and $\beta$ are some elements of $G$ not ending in $y^{-1}$ or $y$ respectively and $k \neq 0$. These two cases are similar so we shall deal only with the first case. We will not spell out such assumptions for the remainder of this proof. Thus $\phi$ is defined by

$$
x \mapsto x \quad y \mapsto\left(\alpha y x^{k}\right) y\left(x^{-k} y^{-1} \alpha^{-1}\right) .
$$

In this paragraph we will show that if $w \notin(x)$ then $|\phi(w)|>|w|$. If $w=$ $x^{a_{1}} y^{b_{1}} x^{a_{2}} y^{b_{2}} \ldots x^{a_{4}} y^{b_{r}}$ then

$$
\phi(w)=x^{a_{1}} \alpha y x^{k}\left(y^{b_{1}}\right) x^{-k} y^{-1} \alpha^{-1} x^{a_{2}} \alpha y x^{k}\left(y^{b_{2}}\right) x^{-k} y^{-1} \alpha^{-1} \ldots x^{a_{1}} \alpha y x^{k}\left(y^{b_{7}}\right) x^{-k} y^{-1} \alpha^{-1} .
$$

When reducing this word the powers of $y$ in parenthesis will never vanish. We may lose some of the original powers of $x$ but only if $\alpha$ begins with a power of $x$. In this case, 
a power of $x$ will be contributed by $\alpha^{-1}$. This shows that $|\phi(w)|>|w|$.

It is clear that if $w \notin\langle x\rangle$ then $\phi(w) \notin\langle x\rangle$. But then we may apply the previous paragraph to such a $w$ to obtain an increasing sequence of integers

$$
|w|<|\phi(w)|<\left|\phi^{2}(w)\right| \ldots<\left|\phi^{r}(w)\right|<\ldots
$$

Thus if $w \notin\langle x\rangle$ and $w \in \phi^{n}(G)$ then $|w|>n$ and so $w \notin \phi^{\infty}(G)$. We conclude that $\phi^{\infty}(G)=\langle x\rangle$ which is obviously a proper free factor of $G$.

Case 2: The map $\phi$ is a type 1 monomorphism, $g \neq 1, h \neq 1$

$$
x \mapsto g x g^{-1} \quad y \mapsto h y h^{-1}
$$

Write $g=\alpha u$ and $h=\alpha v$ so that the product $u^{-1} v$ is reduced: $\alpha$ is the "common initial piece" of $g$ and $h$. If both $u$ and $v$ are nontrivial then it is easy to see that the image of any word $w=x^{a_{1}} y^{b_{1}} x^{a_{2}} y^{b_{2}} \ldots x^{a_{r}} y^{b_{r}}$ grows in length and so $\phi^{\infty}(G)$ is trivial. The situation becomes more complicated if one of $u$ or $v$ is trivial (if both are trivial then $\phi$ is simply an inner automorphism). Without loss of generality we may assume that $u=1$. In this case $\phi$ has the form

$$
x \mapsto \alpha x \alpha^{-1} \quad y \mapsto(\alpha v) y\left(v^{-1} \alpha^{-1}\right) .
$$

If $w$ is written as above then

$$
\phi(w)=\alpha x^{a_{1}} v y^{b_{1}}\left(v^{-1} x^{a_{2}} v\right) y^{b_{2}} v^{-1} \ldots\left(v^{-1} x^{a_{r}} v\right) y^{b_{r}} v^{-1} \alpha^{-1} .
$$

Since the products $v^{-1} x^{a_{i}} v$ for $i=2 \ldots r$ can never be powers of $y$ the only possibility that $|\phi(w)| \leq|w|$ is that cancellation occurs at the beginning or end of this word. Whether such a reduction occurs or not depends on the word $v$. We will show that when $v=x^{-d} \alpha^{-1} x^{d}$ the stable image $\phi^{\infty}(G)$ is nontrivial but in all other cases the stable image is trivial.

Suppose that $v$ is as above so that $\phi$ is defined by

$$
x \mapsto \alpha x \alpha^{-1} \quad y \mapsto\left(\alpha x^{-d} \alpha^{-1} x^{d}\right) y\left(x^{-d} \alpha x^{d} \alpha^{-1}\right) .
$$

Here, the element $x^{d} y x^{-d}$ is fixed so the stable image is clearly nontrivial. To determine its structure we simply make a change of basis for the group $G$. Specifically, let $\bar{x}=x^{d} y x^{-d}$ and $\bar{y}=x$. These elements are generators for the group and if we let $\bar{\alpha}$ denote the word $\alpha$ rewritten in terms of these new generators then $\phi$ is the map

$$
\bar{x} \mapsto \bar{x} \quad \bar{y} \mapsto \bar{\alpha} \bar{y} \bar{\alpha}^{-1} .
$$

This map was studied in case 1 where we showed its stable image was a proper free factor of $G$. 
In this final paragraph we assume $v \neq x^{-d} \alpha^{-1} x^{d}$ for any choice of $d$ and show that $\phi^{\infty}(G)$ is trivial by a length argument. If $w=x^{a_{1}} y^{b_{1}} x^{c_{2}} y^{b_{2}} \ldots x^{a_{1}} y^{b_{r}}$ then

$$
\phi(w)=\alpha x^{a_{1}} v y^{b_{1}}\left(v^{-1} x^{a_{2}} v\right) y^{b_{2}} v^{-1} \ldots\left(v^{-1} x^{a_{1}} v\right) y^{b_{r}} v^{-1} \alpha^{-1} .
$$

It is possible that $|\phi(w)| \leq|w|$ only if $v=x^{t} \alpha^{-1} x^{s}$ for some $t$ and $s$. When $t=-s(\bmod m)$ we have the situation in the previous paragraph. For all other choices of $t$ and $s$ it may be verified that $\left|\phi^{2}(w)\right|>|w|$. Thus for any element $w \in G$ we have an increasing sequence

$$
|w|<\left|\phi^{2}(w)\right|<\left|\phi^{4}(w)\right|<\ldots
$$

This proves that the stable image of $\phi$ is trivial.

Case 3: The map $\phi$ is a type 2 monomorphism

$$
x \mapsto g x g^{-1} \quad y \mapsto h x^{l} h^{-1}
$$

We will not give many details here as the approach is similar to the first two cases. It should be noted that not all type 2 maps are injective but this will not affect our arguments. First, if $g=h$ then $\phi^{\infty}(G)=\left\langle g \times g^{-1}\right\rangle$ and there is nothing to show here. If $g=1$ and $h \neq 1$ then another length argument will show that $w \notin(x)$ implies that $\left|\phi^{2}(w)\right|>|w|$ so that $\phi^{\infty}(G)=\langle x\rangle$. If $g \neq 1$ and $h=1$ then we may as well assume that $g$ ends in a power of $y$. If $g=x^{-r l} y^{r}$ for some $r$ then $y^{r} x y^{-r}$ is fixed and another change of basis argument will revert this case back to the previous one. For all other choices of $g$ the length of the image of a word is bigger than the length of the word and so $\phi^{\infty}(G)$ is trivial.

As before, the most complicated case is when both $g$ and $h$ are nontrivial. We write $g=\alpha u$ and $h=\alpha v$ so that $u^{-1} v$ is reduced. If both $u$ and $v$ are not the identity then $\phi^{\infty}(G)$ is trivial. If $u=1$ and $v \neq 1$ then we may assume that $v$ ends in a power of $y$. This is enough to show that the stable image is trivial by checking that $\left|\phi^{2}(w)\right|>|w|$ for every $w \in G$. Finally, assume $u \neq 1$ and $v=1$. In this case,

$$
x \mapsto \alpha u x u^{-1} \alpha^{-1} \quad y \mapsto \alpha x^{l} \alpha^{-1} .
$$

The situation here is similar to case 2. If $u=x^{-r l} \alpha^{-1} y$ for some $r$ then $y^{\prime} x y^{-r}$ is fixed and it may be shown by a change of basis that $\phi^{\infty}(G)=\left\langle y x y^{-r}\right\rangle$. In all other cases the lengths of words grow under the forward image of $\phi$ so that $\phi^{\infty}(G)$ is trivial.

Corollary 2. The test words for monomorphisms of $G$ are those words of infinite order-i.e., those words not lying in a proper free factor.

Proof. If $w$ lies in a proper free factor then $w$ is a power of a generator $\alpha$ since any proper free factor must be cyclic. Let $\beta$ be a generator of $G$ so that $G$ is equal to the free product $\langle\alpha\rangle *\langle\beta\rangle$. The map 


$$
\alpha \mapsto \alpha \quad \beta \mapsto(\beta \alpha) \beta\left(\alpha^{-1} \beta^{-1}\right)
$$

is a monomorphism since $|\phi(g)| \geq|g|$ for every $g \in G$. Furthermore, $\phi$ is not surjective proving that $w$ is not a test word for monomorphisms.

\section{Test words of $\mathbb{Z}_{m} * \mathbb{Z}_{n}$}

Now that we have determined that the stable image of a monomorphism of $\mathbb{Z}_{m} * \mathbb{Z}_{n}$ is a free factor we may begin to examine the structure of the stable image when $\phi$ is an arbitrary endomorphism. We will not need to use a case by case analysis this time.

Lemma 2. If $G=\left\langle x, y \mid x^{m}, y^{n}\right\rangle$ and $\phi$ is an endomorphism of $G$ then the map $\phi_{\infty}: \phi^{\infty}(G) \rightarrow \phi^{\infty}(G)$ is an automorphism.

Proof. To show that $\phi_{\infty}$ is an automorphism we need only prove that the maps $\phi_{k}: \phi^{k-1}(G) \rightarrow \phi^{k}(G)$ are eventually injective (see Lemma 1). We consider the chain

$$
G \stackrel{\phi_{1}}{\longrightarrow} \phi(G) \stackrel{\phi_{2}}{\longrightarrow} \phi^{2}(G) \rightarrow \ldots \rightarrow \phi^{k-1}(G) \stackrel{\phi_{k}}{\longrightarrow} \phi^{k}(G) \rightarrow \ldots
$$

The subgroups $\phi^{i}(G)$ all have rank less than or equal to 2. By the Kurosh subgroup theorem [2], each subgroup in the chain must be a free product of at most two finite cyclic groups where the ranks on the free factors are divisors of $m$ and $n$. There are only finitely many such groups up to isomorphism so we may choose $N>M$ with $\phi^{N}(G) \cong \phi^{M}(G)$. The composition

$$
\phi^{M}(G) \stackrel{\phi_{M+1}}{\longrightarrow} \phi^{M+1}(G) \rightarrow \ldots \rightarrow \phi^{N-1}(G) \stackrel{\phi_{N}}{\longrightarrow} \phi^{N}(G) \cong \phi^{M}(G)
$$

is a surjective endomorphism of $\phi^{M}(G)$ and so it must be injective as well. This implies that the maps $\phi_{k}, k>M$, are also injective.

Theorem 2. If $G=\mathbb{Z}_{m} * \mathbb{Z}_{n}$ and $\phi$ is an endomorphism of $G$ then the stable image of $\phi$ is a retract of $G$.

Proof. By the proof of Lemma 2 we may choose $M$ so that the map $\phi_{M+1}: \phi^{M}(G) \rightarrow \phi^{M+1}(G)$ is a monomorphism. Regardless of the rank of $\phi^{M}(G)$, the subgroup $\left(\phi_{M+1}\right)^{\infty}\left(\phi^{M}(G)\right)$ is a retract of $\phi^{M}(G)$ (Theorem 1 or the proof of Proposition 1). However, it is clear that $\phi^{\infty}(G)=\left(\phi_{M+1}\right)^{\infty}\left(\phi^{M}(G)\right)$ so if $\rho$ is the retraction mentioned above the composition

$$
G \stackrel{\phi^{M}}{\longrightarrow} \phi^{M}(G) \stackrel{\rho}{\rightarrow} \phi^{\infty}(G) \stackrel{\left(\phi_{\infty}^{-1}\right)^{M}}{\longrightarrow} \phi^{\infty}(G)
$$

is a retraction of $G$ onto the stable image of $\phi$. 
Corollary 3. The test words of $G$ are those not lying in a proper retract.

Example 2. Let $\left.G=\langle x, y| x^{6}, y^{12}\right\}$ and suppose that $\phi$ is the map $x \mapsto x^{3}, y \mapsto y^{4}$. Then $\phi^{\infty}(G)=\phi(G)=\left(x^{3}, y^{4}\right)$. This gives an example of a proper retract of $G$ which is not a proper free factor (since it has rank 2). In particular, the word $x^{3} y^{4}$ is a test word for monomorphisms but not a test word.

\section{Retracts}

In light of Corollary 3 it becomes interesting to determine the structure of the retracts of $\mathbb{Z}_{m} * \mathbb{Z}_{n}$. Example 2 shows that they are not just the free factors of this group.

Suppose that $K$ is a retract of $G=\left\langle x, y \mid x^{m}, y^{n}\right\rangle$ and $\rho: G \rightarrow K$ is a retraction. Then $K$ must have rank less than or equal to 2 . Since we have already described cyclic retracts in general (Proposition 2), we will assume that $K$ has rank 2 for the remainder of this section. Recall that $\rho$ has one of the following forms:
(1) $x \mapsto g x^{k} g^{-1}$
$y \mapsto h y^{\prime} h^{-1}$
(2) $x \mapsto g x^{k} g^{-1}$
$y \mapsto h x^{\prime} h^{-1}$
(3) $x \mapsto g y^{k} g^{-1}$
$y \mapsto h y^{\prime} h^{-1}$
(4) $x \mapsto g y^{k} g^{-1}$
$y \mapsto h x^{\prime} h^{-1}$

Theorem 3. Suppose that $G=\left\langle x, y \mid x^{m}, y^{n}\right\rangle$ and that $K$ is a rank 2 retract of $G$ with retraction $\rho$. Then $\rho$ is type 1,2 , or 3 .

(a) If $\rho$ is a type 1 retraction then $K=\left\langle g x^{k} g^{-1}, h y^{\prime} h^{-1}\right)$ such that

(1) $k^{2}=k(\bmod m)$ and $l^{2}=l(\bmod n)$, and

(2) $g, h \in\left\langle\left\langle x^{s}, y^{t}\right\rangle\right)$, the normal subgroup generated by $x^{s}$ and $y^{t}$, where $s$ (resp. $\left.t\right)$ is the order of $x^{k}$ (resp. $\left.y^{l}\right)$.

(b) If $\rho$ is a type 2 retraction then $K=\left\langle g x^{k} g^{-1}, h x^{l} h^{-1}\right\rangle$ such that

(3) $k^{2}=k(\bmod m)$ and $k l=l(\bmod m)$, and

(4) $g \in\left\langle\left(x^{s}, y^{t}\right\rangle\right.$ where $s$ (resp. $\left.t\right)$ is the order of $x^{k}$ (resp. $\left.x^{t}\right)$.

(c) If $\rho$ is a type 3 retraction then $K=\left\langle g y^{k} g^{-1}, h y^{l} h^{-1}\right\rangle$ such that

(5) $k l=k(\bmod n)$ and $l^{2}=l(\bmod n)$, and

(6) $h \in\left\langle\left(x^{s}, y^{t}\right\rangle\right.$ where $s$ (resp. $\left.t\right)$ is the order of $y^{k}\left(\right.$ resp. $\left.y^{t}\right)$.

Proof. Since $\rho^{2}=\rho$, this map is clearly not type 4. Therefore, first suppose that $\rho$ is a type 1 retraction given by

$$
x \mapsto g x^{k} g^{-1} \quad y \mapsto h y^{\prime} h^{-1} .
$$

Again, since $\rho^{2}=\rho$ we have the equation

$$
\rho(g) g x^{k^{2}} g^{-1} \rho(g)^{-1}=g x^{k} g^{-1}
$$


and (1) clearly holds by abelianizing $G$. Replacing $k^{2}$ with $k$ we see that

$$
\left(g^{-1} \rho(g) g\right) x^{k}=x^{k}\left(g^{-1} \rho(g) g\right)
$$

and a standard result of free products [3] states that the elements $g^{-1} \rho(g) g$ and $x^{k}$ are in the same conjugate of a free factor of $G$ or are both powers of the same element $w \in G$. In either case $g^{-1} \rho(g) g$ is some power of $x$. Our map $\rho$ now looks like

$$
x \mapsto\left(\rho(g)^{-1} g x^{d}\right) x^{k}\left(x^{-d} g^{-1} \rho(g)\right)=\left(\rho(g)^{-1} g\right) x^{k}\left(g^{-1} \rho(g)\right)
$$

and the conjugator may be assumed to be an element of the kernel. Hence $\rho$ is a map defined by

$$
x \mapsto g^{\prime} x^{k}\left(g^{\prime}\right)^{-1} \quad y \mapsto h^{\prime} y^{l}\left(h^{\prime}\right)^{-1}
$$

where $g^{\prime}, h^{\prime} \in \operatorname{ker}(\rho)$.

We will now show that the kernel of $\rho$ is normally generated by elements $x^{s}$ and $y^{t}$ as outlined in (2). If $s$ and $t$ are as stated then it is clear that $\left\langle\left\langle x^{s}, y^{t}\right\rangle\right\rangle$ is a normal subgroup of $\operatorname{ker}(\rho)$. We will prove that $\operatorname{ker}(\rho) /\left\langle\left\langle x^{s}, y^{t}\right\rangle\right.$ is trivial. By Kurosh, $\rho(G) \cong\left\langle g x^{k} g^{-1}\right\rangle *\left\langle h y^{l} h^{-1}\right\rangle \cong \mathbb{Z}_{s} * \mathbb{Z}_{t}$ and by the Noether Isomorphism Theorems, $\left.\rho(G) \cong G /\left\langle\left\langle x^{s}, y^{t}\right\rangle\right\rangle / \operatorname{ker}(\rho) /\left\langle x^{s}, y^{t}\right\rangle\right\rangle$. Furthermore, $G /\left\langle\left\langle x^{s}, y^{t}\right\rangle\right\rangle=\left\langle x, y \mid x^{m}, y^{n}, x^{s}, y^{t}\right\rangle \cong \mathbb{Z}_{s} * \mathbb{Z}_{t}$ which finishes the proof in this case.

Now suppose that $\rho$ is a type 2 retraction given by

$$
x \mapsto g x^{k} g^{-1} \quad y \mapsto h x^{t} h^{-1} .
$$

The proof we give here will obviously work in the case that $\rho$ is a type 3 retraction. Item (3) holds by previous arguments. The element $g$ can be assumed to be an element of $\operatorname{ker}(\rho)$ as before. Finally, if $g \neq h$ then

$$
K \cong\left(g x^{k} g^{-1}\right) *\left(h x^{\prime} h^{-1}\right) \cong \mathbb{Z}_{s} * \mathbb{Z}_{t}
$$

and the proof of (2) also holds for (4). If $g=h$ then $K$ is cyclically generated by the element $g x^{d} g^{-1}$ where $d=g c d(k, l)$ which cannot happen since $K$ has rank 2 .

Example 3. The element $w=x y$ is a test word of $\mathbb{Z}_{m} * \mathbb{Z}_{n}$.

It is evident that $x y$ cannot lie in a cyclic retract or a rank 2 retract of type 2 or type 3. If $x y$ is an element of a type 1 retract $K=\left\langle g x^{k} g^{-1}, h y^{\prime} h^{-1}\right\rangle$ where $k^{2}=k(\bmod m)$ and $l^{2}=l(\bmod n)$ then $k=l=1$. This is easily seen by abelianizing $G$ to get the equations $k d_{1}=1(\bmod m)$ and $l d_{2}=1(\bmod n)$ for some $d_{i}$. By Theorem 3 , the conjugators $g, h \in\left\langle\left\langle x^{m}, y^{n}\right\rangle\right)$ which implies that $K$ is not proper.

Example 4. The commutator $[x, y]$ is a test word of $\mathbb{Z}_{m} * \mathbb{Z}_{n}$. 
Every nontrivial element of a cyclic retract $\left(g x^{k} g^{-1}\right)$ has nonzero exponent sum on $x$ so it is impossible for such a retract to contain the commutator element.

Suppose

$$
\rho: G \rightarrow K=\left\langle g x^{k} g^{-1}, h x^{l} h^{-1}\right\rangle
$$

is a type 2 retraction and that $[x, y] \in K$. Then the image of $[x, y]$ is fixed under $\rho$ so that

$$
x y x^{-1} y^{-1}=g x^{k}\left(g^{-1} h\right) x^{l}\left(h^{-1} g\right) x^{-k}\left(g^{-1} h\right) x^{-1} h^{-1} .
$$

If the right hand side of this equation has length 4 then $g^{-1} h$ must be a power of $x$. But then $K$ would be cyclic and we have dealt with this case already. Clearly type 3 retractions may be disposed of in the same manner.

Finally, suppose

$$
\rho: G \rightarrow K=\left\langle g x^{k} g^{-1}, h y^{l} h^{-1}\right\rangle
$$

is a type 1 retraction. In case 2 of Theorem 1 we dealt with similar maps where $k=l=1$. It was shown there that such maps had proper nontrivial stable images if and only if $h=\left[g, x^{d}\right]$, or symmetrically, $g=\left[h, y^{d}\right]$ for some $d$. The same arguments apply here for the map $\rho$. But any non-identity map of the form

$$
x \mapsto g x^{k} g^{-1} \quad y \mapsto\left[g, x^{d}\right] y^{l}\left[g, x^{d}\right]^{-1}
$$

can never fix the commutator, proving that $[x, y]$ does not lie in $K$.

Example 5. The element $w=x^{k} y^{k}(k>1)$ is not a test word of $\mathbb{Z}_{m} * \mathbb{Z}_{n}$ for certain $m$ and $n$.

The endomorphism of the group $\left.\langle x| x^{10}\right)$ which maps $x$ to $x^{6}$ is a retract onto $\left\langle x^{6}\right\rangle$. Thus $x^{2} y^{2}$ lies in a proper retract of $\left(x, y \mid x^{10}, y^{10}\right)$. If $k \geq 3$ then choose $m=k^{2}-k$. In this case, the subgroup $\left\langle x^{k}, y^{k}\right\rangle$ is a proper retract of $\left\langle x, y \mid x^{m}, y^{m}\right\rangle$ containing $x^{k} y^{k}$.

Example 6. The test words of $\mathbb{Z}_{p_{1}^{m}} * \mathbb{Z}_{p_{2}^{\prime \prime}}\left(p_{1} \neq p_{2}\right.$ are primes) coincide with the test words for monomorphisms.

Suppose $x$ and $y$ are generators for $\mathbb{Z}_{p_{1}^{m}} * \mathbb{Z}_{p_{2}^{n}}$. We need to show that the retracts of this group are precisely the free factors. Any endomorphisms of this group must be a type 1 map so any retract is a type 1 retract. Let $H$ be a nontrivial proper retract of $\mathbb{Z}_{p_{i}^{k}} * \mathbb{Z}_{p^{k}}$ generated by the elements $g x^{k} g^{-1}$ and $h y^{\prime} h^{-1}$ for some $k$ and $l$. The equation $k^{1}=k\left(\bmod p_{1}^{m}\right)$ implies that $k=0,1\left(\bmod p_{1}^{m}\right)$. Similarly, $l=0,1\left(\bmod p_{2}^{n}\right)$. Since $H$ is a nontrivial subgroup, one of $k$ or $l$ is zero but not both. In particular, assuming $k=0$, $H=\left(h y h^{-1}\right)$ and is a proper free factor. 
Example 7. $P S L(2, \mathbb{Z})$

The special linear group $S L(2, \mathbb{Z})$, of $2 \times 2$ integral matrices with determinant 1 is generated by the elements

$$
x=\left(\begin{array}{rr}
0 & 1 \\
-1 & 0
\end{array}\right) \text { and } y=\left(\begin{array}{rr}
0 & 1 \\
-1 & 1
\end{array}\right) .
$$

The modular group $\operatorname{PSL}(2, \mathbb{Z})$ is the quotient of $S L(2, \mathbb{Z})$ by $\left\langle x^{2}\right\rangle$ and has the presentation

$$
\operatorname{PSL}(2, \mathbb{Z})=\left\langle x, y \mid x^{2}, y^{3}\right\rangle .
$$

It follows that this group contains the test words

$$
x y=\left(\begin{array}{rr}
-1 & 1 \\
0 & -1
\end{array}\right) \text { and }[x, y]=\left(\begin{array}{rr}
2 & -1 \\
-1 & 1
\end{array}\right)
$$

\section{REFERENCES}

1. W. IMRICH and E. C. TURNER, Endomorphisms of free groups and their fixed points, Math. Proc. Cambridge Philos. Soc 105 (1989), 421-422.

2. R. LyNDON and P. SCHUPP, Combinatorial Group Theory (Springer-Verlag, 1970).

3. W. Magnus, A. Karrass and D. Solitar, Combinatorial Group Theory (Wiley, New York, 1966).

4. E. C. TURNER, Test words for automorphisms of free groups, Bull. London Math. Soc., to appear.

Department of Mathematics and Statistics

UNIVERSITY AT ALBANY

Albany, New York 12222

USA 